\title{
The flavor problem and discrete symmetries
}

\author{
A. C. B. Machado ${ }^{1}$, V. Pleitez ${ }^{2}$ \\ Instituto de Física Teórica, Universidade Estadual Paulista \\ Rua Pamplona 145, 01405-900 - São Paulo, SP, Brazil
}

\begin{abstract}
In this Letter we propose a multi-Higgs extension of the standard model with Abelian and non-Abelian discrete symmetries in which the mass matrices of the charged fermions obtained from renormalizable interactions are diagonal. However, non-diagonal contributions, that are important for obtaining the CKM matrix in the quark sector, arise from non-renormalizable dimension five interactions. Active neutrinos acquire mass only from non-renormalizable interactions, the non-diagonal entries arising through dimension five operators, while the diagonal entries comes from dimension six operators. Realistic mixing matrices in the neutrino and the quarks sectors are obtained.
\end{abstract}

Key words: multi-Higgs models, $A_{4}$ symmetry, quark and lepton masses and mixing.

PACS: 11.30.Hv; 12.60.Fr; 12.15.Ff; 14.60.Pq

The flavor problem can be divided in several subproblems [1]: Why do weak isospin partners have different masses? Why are quark and lepton masses split? Why is there a mass hierarchy between generations? Presently we know that neutrino oscillation data are well described by massive neutrinos [2] and the flavor problem becomes more interesting: why is there a mixing angle hierarchy in the quark sector but not in the lepton sector? Usually mass matrices have the form $M_{\alpha \beta}=\sum_{i}\left(\Gamma_{i}\right)_{\alpha \beta}\left\langle\Phi_{i}^{0}\right\rangle$, where the $\Gamma_{i}$ 's are, for Dirac fermions, arbitrary complex dimensionless $3 \times 3$ matrices, and $\left\langle\Phi_{i}^{0}\right\rangle$ denotes one or a set of vacuum expectation values $(\mathrm{VEVs})$ of the neutral scalar fields in the model. For Majorana fermions, the $\Gamma_{i}$ 's are complex symmetric matrices. The mixing matrix and the mass pattern of each charged sector depend on the structure of the respective $\Gamma_{i}$ 's. It is well known that explicit and predictive

\footnotetext{
Email addresses: ana@ift.unesp.br (A. C. B. Machado), vicente@ift.unesp.br (V. Pleitez).

1 Supported by CNPq

2 Partially supported by CNPq under the process 300613/2005-9
} 
forms of these matrices can be obtained by imposing flavor symmetries. For instance, it has been considered global unitary (horizontal) symmetries like $U(1)^{\text {'s, }} S U(2)_{H}$ [3] or $S U(3)_{H}$ [4]. More recently symmetries like $S_{n}, Z_{n}$ [5]; $A_{4}$ [6], ${ }^{(d)} T$ [7,8], the double covering of $A_{4} ; Q_{6}$, the double dihedral group [9], and other discrete symmetries [10] have been also considered.

Here we will turn the problem upside down. Mass matrices will be of the form $M_{\alpha \beta}=f\left(\overline{\left\langle\Phi^{0}\right\rangle}\right)_{\alpha \beta}$ in which $f$ denotes a few dimensionless $(\mathcal{O}(1))$ parameters (one at leading order), and $\overline{\left\langle\Phi^{0}\right\rangle}$ is a matrix built with the VEVs of several scalar fields. At first sight, there is no gain in predictive power, we are just changing a dimensionless general matrix $\Gamma_{\alpha \beta}$ by another one with mass dimension $\left(\overline{\left\langle\Phi^{0}\right\rangle}\right)_{\alpha \beta}$. However, it seems easier, at least in principle, to explain patterns of dynamical variables like VEVs, than dimensionless numbers. The value of the former can be explained by the dynamics (for instance by studying the scalar potential) and extra flavor symmetries that we can impose to the model.

In this Letter we will consider an extension of the standard model in which the charged fermion masses, that arise from renormalizable interactions, are diagonal. However, dimension 5 operators [11] give important non-diagonal contributions to quarks but not to the charged leptons. On the other hand, the neutrinos mass matrix is generated mainly from dimension 5 and 6 operators, with the former having zero trace as in the Zee's mode [12]. The dimension 6 operators induce non-negligible diagonal terms and a general $3 \times 3$ symmetric mass matrix for those neutrinos is obtained. The non-diagonal elements are important in the quark sector because they involve also the VEV of the singlet which it is not constrained by the electroweak scale, and also in the neutrino sector because theses entries involve ratios of doublets' VEVs that produce an enhancement. This enhancement does not occurs in the charged lepton sector because in this case the non-diagonal entries depend on the same doublet's VEVs that appear in the diagonal ones but are suppressed by $\Lambda^{-1}$. No righthanded neutrinos are introduced. In summary, we show that all the questions mentioned above have a natural answer: fermion masses and mixing arise from different scalar sectors and from different effective interactions. The predictive power is a consequence of the discrete symmetries imposed to the model.

Simple non-Abelian discrete groups have been considered for the first time as candidates for flavor symmetries by Frampton and Kephart [7]. Later, $A_{4}$ symmetry has been used to obtain realistic mixing matrices in the lepton sector [13], and then also in the quark sectors [14]. More recently [15], by using these non-Abelian discrete symmetries it was possible to obtain a tribimaximal neutrino mixing matrix [16]. The basic idea is to consider $S U(2)_{L}$ fermion doublets and singlets in the triplet representation of the $A_{4}$ symmetry and Higgs multiplets transforming as triplet or singlet of $A_{4}$. 
Let us briefly review some properties of the $A_{4}$ representations. Denoting the vector basis $(\mathbf{3})$ as $\left(a_{1}, a_{2}, a_{3}\right)$ and $\left(b_{1}, b_{2}, b_{3}\right)$ we have $\mathbf{3} \otimes \mathbf{3}=\mathbf{3}_{A} \oplus \mathbf{3}_{B} \oplus \mathbf{1} \oplus$ $\mathbf{1}^{\prime} \oplus \mathbf{1}^{\prime \prime}$. Explicitly [17],

$$
\begin{aligned}
& {[\mathbf{3} \otimes \mathbf{3}]_{A} \equiv \mathbf{3}_{A}=\left(a_{2} b_{3}, a_{3} b_{1}, a_{1} b_{2}\right), \quad[\mathbf{3} \otimes \mathbf{3}]_{B} \equiv \mathbf{3}_{B}=\left(a_{3} b_{2}, a_{1} b_{3}, a_{2} b_{1}\right),} \\
& {[\mathbf{3} \otimes \mathbf{3}]_{1}=a_{1} b_{1}+a_{2} b_{2}+a_{3} b_{3}, \quad[\mathbf{3} \otimes \mathbf{3}]_{1^{\prime}}=a_{1} b_{1}+\omega a_{2} b_{2}+\omega^{2} a_{3} b_{3},} \\
& {[\mathbf{3} \otimes \mathbf{3}]_{1^{\prime \prime}}=a_{1} b_{1}+\omega^{2} a_{2} b_{2}+\omega a_{3} b_{3},}
\end{aligned}
$$

and $1 \otimes 1=1,1^{\prime} \otimes 1^{\prime \prime}=1,1^{\prime} \otimes 1^{\prime}=1^{\prime \prime}, 1^{\prime \prime} \otimes 1^{\prime \prime}=1^{\prime}$.

We consider a model with $G_{S M} \otimes A_{4} \otimes Z_{3} \otimes Z_{3}^{\prime} \otimes Z_{3}^{\prime \prime}$ symmetry, where $G_{S M}$ is the gauge symmetry $S U(3)_{C} \otimes S U(2)_{L} \otimes U(1)_{Y}$. The matter fields transform as usual under $G_{S M}$, left-handed lepton and quark doublets $L_{a}, a=e, \mu, \tau$; $Q_{i}, i=1,2,3$, and their respective right-handed singlets $l_{a R}, u_{i R}$ and $d_{i R}$. In the scalar sector, we introduce scalar doublets with $Y=+1: H_{i}, \hat{H}_{i}, H_{i}^{\prime}, H_{i}^{\prime \prime}$, $\Phi_{i}, \Phi_{i}^{\prime}, \Phi_{i}^{\prime \prime}$ and $\chi$; three scalar triplets with $Y=+2$, denoted by $\mathcal{T}_{i}$ [18], and finally one complex (with $Y=0$ ) scalar singlet $\zeta$. Fermion weak eigenstates fields transform under $\left(A_{4}, Z_{3}, Z_{3}^{\prime}, Z_{3}^{\prime \prime}\right)$ as follows:

$$
\begin{aligned}
& L \equiv\left(L_{e}, L_{\mu}, L_{\tau}\right) \sim\left(\mathbf{3}, \omega, \omega^{2}, 1\right) ; \quad l_{R} \equiv\left(\mu_{R}, \tau_{R}, e_{R}\right) \sim(\mathbf{3}, 1,1, \omega), \\
& Q_{L} \equiv\left(Q_{1 L}, Q_{2 L}, Q_{3 L}\right) \sim\left(\mathbf{3}, \omega^{2}, 1,1\right) ; \quad u_{R} \equiv\left(c_{R}, t_{R}, u_{R}\right) \sim(\mathbf{3}, 1,1, \omega) ; \\
& d_{R} \equiv\left(s_{R}, b_{R}, d_{R}\right) \sim(3,1, \omega, \omega),
\end{aligned}
$$

in which $\omega=e^{2 \pi i / 3}$. Notice that all fermion fields are still symmetry eigenstates but we do not use an special notation. On the other hand, scalar fields transform under $\left(A_{4}, Z_{3}, Z_{3}^{\prime}, Z_{3}^{\prime \prime}\right)$ as:

$$
\begin{aligned}
& H \equiv\left(H_{1}, H_{2}, H_{3}\right) \sim\left(\mathbf{3}, \omega, \omega^{2}, 1\right), \hat{H} \equiv\left(\hat{H}_{1}, \hat{H}_{2}, \hat{H}_{3}\right) \sim\left(\mathbf{3}, \omega, \omega^{2}, \omega^{2}\right) \\
& H^{\prime} \equiv\left(H_{1}^{\prime}, H_{2}^{\prime}, H_{3}^{\prime}\right) \sim(\mathbf{3}, \omega, 1, \omega), H^{\prime \prime} \equiv\left(H_{1}^{\prime \prime}, H_{2}^{\prime \prime}, H_{3}^{\prime \prime}\right) \sim\left(\mathbf{3}, \omega^{2}, \omega^{2}, \omega^{2}\right) \\
& \Phi \equiv\left(\Phi_{1}, \Phi_{2}, \Phi_{3}\right) \sim(\mathbf{3}, 1,1,1), \Phi^{\prime} \equiv\left(\Phi_{1}^{\prime}, \Phi_{2}^{\prime}, \Phi_{3}^{\prime}\right) \sim(\mathbf{3}, \omega, \omega, \omega) \\
& \Phi^{\prime \prime} \equiv\left(\Phi_{1}^{\prime \prime}, \Phi_{2}^{\prime \prime}, \Phi_{3}^{\prime \prime}\right) \sim\left(\mathbf{3}, \omega^{2}, 1, \omega^{2}\right), \chi \sim\left(\mathbf{1}, \omega^{2}, \omega, 1\right) \\
& \mathcal{T} \equiv\left(\mathcal{T}_{1}, \mathcal{T}_{2}, \mathcal{T}_{3}\right) \sim\left(\mathbf{3}, \omega^{2}, \omega, 1\right), \zeta \sim(\mathbf{1}, 1, \omega, 1)
\end{aligned}
$$

With the fields in (21) and (3) we have the leading contributions to the lepton and quark Yukawa interactions:

$$
\begin{aligned}
\mathcal{L} & =\frac{1}{\Lambda}\left(f_{\nu}\left[\left(\overline{L^{c}} \epsilon H\right)\right]_{A}[(L \epsilon \Phi)]_{B}+\frac{f_{\nu}^{\prime}}{\Lambda}\left[\overline{L^{c}} \varepsilon \vec{\sigma} \cdot \overrightarrow{\mathcal{T}}\right]_{B}\left[L \Phi^{\dagger}\right]_{B} \chi+\ldots\right)+\text { H.c. } \\
& +\left(g_{l}[\bar{L} \hat{H}]_{A}+\frac{g_{l}^{\prime}}{\Lambda^{2}}[\bar{L} \hat{H}]_{A}|\zeta|^{2}+\cdots\right) l_{R}+\text { H.c. }
\end{aligned}
$$




$$
\begin{aligned}
& +\left(h_{u}\left[\overline{Q_{L}} \widetilde{H}^{\prime}\right]_{A}+\frac{h_{u}^{\prime}}{\Lambda}\left[\overline{Q_{L}} \widetilde{\Phi}^{\prime}\right]_{B} \zeta+\cdots\right) u_{R}+H . c . \\
& +\left(h_{d}\left[\overline{Q_{L}} H^{\prime \prime}\right]_{A}+\frac{h_{d}^{\prime}}{\Lambda}\left[\overline{Q_{L}} \Phi^{\prime \prime}\right]_{B} \zeta^{*}+\cdots\right) d_{R}+H . c .
\end{aligned}
$$

where $\Lambda$ is an energy scale characterizing an unknown physics; and $\varepsilon=i \sigma_{2}$ ( $\sigma_{2}$ being the Pauli matrix), [ ] $]_{A, B}$ means the appropriate product defined in Eq. (11) and $\cdots$ denotes higher-dimensional operators, we assume that $|V| \ll \Lambda$, where $V$ denotes the VEV of any of the scalar of the model.

The contributions to the quark mass matrices obtained from renormalizable interactions are diagonal, but the non-renormalizable dimension 5 interactions contribute with important and not negligible non-diagonal elements, on the other hand, for the charged leptons this corrections come from dimension 6 operators are negligible. We obtain diagonal entries to the mass matrices as a consequence of the definition of the basis in which the product $\left[[]_{A} \cdot\right]_{1}$ gives the diagonal contribution while the product $\left[[]_{B} \cdot\right]_{1}$ gives the non-diagonal contributions to the mass matrix. For instance, for the $u$-quarks, the leading diagonal mass matrix arises through the interaction $\left[\bar{Q} H^{\prime}\right]_{A}=\left(\bar{Q}_{2} H_{3}^{\prime}, \bar{Q}_{3} H_{1}^{\prime}, \bar{Q}_{1} H_{2}^{\prime}\right)$ and then $\left[\left[\bar{Q} H^{\prime}\right]_{A}\left(c_{R}, t_{R}, u_{R}\right)\right]_{1}=\bar{Q}_{2} H_{3}^{\prime} c_{R}+\bar{Q}_{3} H_{1}^{\prime} t_{R}+\bar{Q}_{1} H_{2}^{\prime} u_{R}$. Similarly, using the []$_{B}$ product, we obtain that $\left[\left[\bar{Q} \Phi^{\prime}\right]_{B}\left(c_{R}, t_{R}, u_{R}\right)\right]_{1}=\bar{Q}_{3} \Phi_{2}^{\prime} c_{R}+\bar{Q}_{1} \Phi_{3}^{\prime} t_{R}+$ $\bar{Q}_{2} \Phi_{1}^{\prime} u_{R}$, which gives the non-diagonal contributions to the charged lepton mass matrix.

Hence, from the Yukawa interactions in Eq. (4), the mass matrices obtained are

$$
M^{l} \approx g_{l}\left(\begin{array}{ccc}
\hat{v}_{2} & 0 & 0 \\
0 & \hat{v}_{3} & 0 \\
0 & 0 & \hat{v}_{1}
\end{array}\right)+H . c .
$$

for the charged leptons. We have denoted $\left\langle\hat{H}_{i}\right\rangle=\hat{v}_{i}$. Notice that the renormalizable interactions are the dominant one, i.e., $g_{l} \hat{v}_{1} \simeq m_{\tau}, g_{l} \hat{v}_{3} \simeq m_{\mu}$ and $g_{l} \hat{v}_{2} \simeq m_{e}$. In the quark sector we have

$$
M^{u} \approx h_{u}\left(\begin{array}{ccc}
v_{2}^{\prime} & 0 & a_{u} v_{\phi_{3}}^{\prime} \\
a_{u} v_{\phi_{1}}^{\prime} & v_{3}^{\prime} & 0 \\
0 & a_{u} v_{\phi_{2}}^{\prime} & v_{1}^{\prime}
\end{array}\right)+H . c .
$$


and

$$
M^{d} \approx h_{d}\left(\begin{array}{ccc}
v_{2}^{\prime \prime} & 0 & a_{d} v_{\phi_{3}}^{\prime \prime} \\
a_{d} v_{\phi_{1}}^{\prime \prime} & v_{3}^{\prime \prime} & 0 \\
0 & a_{d} v_{\phi_{2}}^{\prime \prime} & v_{1}^{\prime \prime}
\end{array}\right)+H . c .
$$

in which $a_{u}=\frac{h_{u}^{\prime}}{h_{u}} \frac{v_{\zeta}}{\Lambda}$ and $a_{d}=\frac{h_{d}^{\prime}}{h_{d}} \frac{v_{\zeta}}{\Lambda}$, for the $2 / 3$ and $-1 / 3$ charged quarks, respectively. We have denoted $\left\langle H_{i}^{0}\right\rangle=v_{i},\left\langle H_{i}^{\prime 0}\right\rangle=v_{i}^{\prime},\left\langle H_{i}^{\prime \prime 0}\right\rangle=v_{i}^{\prime \prime},\left\langle\Phi_{i}\right\rangle=v_{\phi_{i}}$, $\left\langle\Phi_{i}^{\prime}\right\rangle=v_{\phi_{i}}^{\prime},\left\langle\Phi_{i}^{\prime \prime}\right\rangle=v_{\phi_{i}}^{\prime \prime}$, and $\langle\zeta\rangle=v_{\zeta}$. Notice, as well, that each charged sector has its private VEVs, thus all these matrices are independent from each other. In the case of neutrinos we have the mass matrix

$$
M^{\nu} \approx\left[\left(\begin{array}{ccc}
0 & \frac{v_{2}}{v_{1}} & \frac{v_{\phi_{3}}}{v_{\phi_{1}}} \\
\frac{v_{2}}{v_{1}} & 0 & \frac{v_{3}}{v_{1}} \frac{v_{\phi_{2}}}{v_{\phi_{1}}} \\
\frac{v_{\phi_{3}}}{v_{\phi_{1}}} & \frac{v_{3}}{v_{1}} \frac{v_{\phi_{2}}}{v_{\phi_{1}}} & 0
\end{array}\right)+\left(\begin{array}{ccc}
\delta_{1} & 0 & 0 \\
0 & \delta_{2} & 0 \\
0 & 0 & \delta_{3}
\end{array}\right)\right] \frac{f_{\nu} v_{\phi_{1}}}{\Lambda} v_{1},
$$

where the $\delta_{i}$ are given by $\delta_{1}=\frac{f_{\nu}^{\prime}}{f_{\nu}}\left(v_{T 3} v_{\phi_{3}} v_{\chi} / \Lambda v_{1} v_{\phi_{1}}\right), \delta_{2}=\frac{f_{\nu}^{\prime}}{f_{\nu}}\left(v_{T 1} v_{\chi} / \Lambda v_{1}\right)$, and $\delta_{3}=\frac{f_{\nu}^{\prime}}{f_{\nu}}\left(v_{T 2} v_{\phi_{2}} v_{\chi} / \Lambda v_{1} v_{\phi_{1}}\right)$, where $\langle\chi\rangle=v_{\chi}$, and $\left\langle\Delta_{i}^{0}\right\rangle=v_{T i}$.

It is interesting that the discrete symmetries of the model implies that the charged lepton mass matrix, Eq. (5), is practically diagonal, and that the quark mass matrices have three zeros, see Eqs. (6) and (17). To the best of our knowledge, this mass matrices have not been considered in literature [19]. Notice that in the case of the neutrino we have already taken into account the hermitian conjugate.

Since the only constraint on the VEVs of the doublets is that $\sum v^{2}<(174 \mathrm{GeV})^{2}$ (there are triplets as well, see below), a possibility is that only $v_{1}^{\prime 2} \lesssim(174 \mathrm{GeV})^{2}$, while all the other VEVs are $\ll 174 \mathrm{GeV}$. In this case $v_{1}^{\prime}$ give the main contribution to the mass of the $t$ quark. On the other hand, the sum of the VEVs of the triplets has to have a upper limit of the order of a few $\mathrm{GeV}$ to not spoil the observed value of the $\rho$ parameter [20]. Hence, $|V| / \Lambda$ are well-defined expansion parameters. The present model is natural in the Yukawa interactions i.e., unlike the standard model, there is no a hierarchy among the Yukawa couplings. In practice, in the analysis below we will consider that all Yukawa coupling are in the interval $0.1-3$. The VEV of the singlet $v_{\zeta}$ is only constrained by the condition $v_{\zeta} / \Lambda<1$. Below we will use always, just as an illustration: $\Lambda=1 \mathrm{TeV}$ and $v_{\zeta}=140 \mathrm{GeV}$. However, recall that the singlet $\zeta$ may be related to a new energy scale different from the electroweak scale.

As a first illustration, let us consider the matrix in Eq. (8). We will as- 
sume, without losing generality, that this matrix is a traceless matrix i.e., that the condition $\sum_{i} \delta_{i}=0$ is valid [12]. This is obtained by imposing (using $\tilde{v}_{x}=v_{x} e^{i \theta_{x}}$, where $v_{x}$ and $\theta_{x}$ are real):

$$
\begin{aligned}
& \theta_{T 3}+\theta_{\phi_{3}}-\theta_{1}-\theta_{\phi_{1}}=\pi, \quad \theta_{T 1}-\theta_{1}=0 ; \quad \theta_{T 2}+\theta_{\phi_{1}}-\theta_{1}-\theta_{\phi_{1}}=0 \\
& (1 / 2)\left|\frac{f_{\nu}^{\prime}}{f_{\nu}} \frac{v_{T_{3}} v_{\chi} v_{\phi_{3}}}{\Lambda v_{1} v_{\phi_{1}}}\right|=\left|\frac{f_{\nu}^{\prime}}{f_{\nu}} \frac{v_{T_{1}} v_{\chi}}{\Lambda v_{1}}\right|=\left|\frac{f_{\nu}^{\prime}}{f_{\nu}} \frac{v_{T_{2}} v_{\chi} v_{\phi_{2}}}{\Lambda v_{1} v_{\phi_{1}}}\right| \equiv \delta
\end{aligned}
$$

Hence, the neutrino mass matrix is of the form [21,22]

$$
M^{\nu} \approx m_{0}\left(\begin{array}{ccc}
-2 \delta & \sin \theta & \cos \theta \\
\sin \theta & \delta & \epsilon \\
\cos \theta & \epsilon & \delta
\end{array}\right)
$$

where $m_{0}=\left(f_{\nu} / \Lambda\right)\left|v_{\phi_{1}} v_{1}\right|,\left|v_{2} / v_{1}\right|=\sin \theta,\left|v_{\phi_{3}} / v_{\phi_{1}}\right|=\cos \theta, \epsilon=\left|\left(v_{3} v_{\phi_{2}} / v_{1} v_{\phi_{1}}\right)\right|$. From Table I of Ref. [22] we can see that neutrinos masses and mixing angles are in agreement with all experimental data if, for instance, in Eq. (10), $m_{0}^{2}=\Delta m_{13}^{2} \equiv \Delta m_{a t m}^{2}=(0.039 \mathrm{eV})^{2}, \epsilon=0.21, \delta=0.236$, and $\theta=0.84$. In our model, these values for the above parameters are obtained by assuming: $\Lambda=1$ $\mathrm{TeV}, v_{1} \sim 1 \mathrm{MeV}, v_{\phi_{1}}=v_{\phi_{2}}, 2 v_{T_{1}}=2 v_{T_{2}}=v_{T_{3}}$. with $v_{T_{1}}=1.25 \mathrm{GeV}$; then we obtain: $f_{\nu} v_{\phi_{1}} \sim 40 \mathrm{keV}, v_{\phi_{3}}=40 / f_{\nu} \mathrm{keV}, v_{2}=0.1 \mathrm{keV}, v_{3}=0.2 \mathrm{MeV}$, $\left(f_{\nu} / f_{\nu}^{\prime}\right) v_{\chi}=0.2 \mathrm{GeV}$. We also obtain with these VEVs, $\Delta m_{12}^{2}=9 \times 10^{-5} \mathrm{eV}^{2}$, $\sin ^{2} 2 \theta_{\odot}=0.82, \sin ^{2} 2 \theta_{a t m}=0.99$, and $\sin \theta_{13}=0.019$ a value that is in agreement with the CHOOZ limit $V_{e 3}=\sin \theta_{13}<0.07$ [23]. We see that a realistic leptonic mixing matrix arises since the mass matrix of the charged leptons is almost diagonal. If $f_{\nu}, f_{\nu}^{\prime} \sim \mathcal{O}(1)$, it implies that some VEVs are of the order of $\mathrm{MeV}$ or even less depending on the values of $v_{\zeta}$ and $\Lambda$. This does not necessarily implies the existence of light scalars since for having them in a model, there must be some symmetries that allow them to appear in the mass spectra: The symmetries in the present model do not play that role and $\mu_{i j}^{2}$ terms survive in the scalar mass matrices (see Ref. [24]).

On the other hand, with $M^{u, d}$ given by Eq. (6) and (7) we see that $M^{u(d)} M^{u(d) \dagger} \neq$ $M^{u(d) \dagger} M^{u(d)}$. Hence we need four matrices, $V_{L, R}^{U, D}$, for diagonalizing these mass matrices by biunitary transformations and the constraint $V_{C K M}=V_{L}^{U} V_{L}^{D \dagger}$. Besides, the matrices $V_{L, R}^{U, D}$ must satisfy the constraints $V_{L}^{D} M^{d} M^{d \dagger} V_{L}^{D \dagger}=$ $\operatorname{diag}\left(m_{d}^{2}, m_{s}^{2}, m_{b}^{2}\right)=\left(\hat{M}^{d}\right)^{2}$ and $V_{R}^{D} M^{d \dagger} M^{d} V_{R}^{D \dagger}=\left(\hat{M}^{d}\right)^{2}$; similarly for the $V_{L, R}^{U}$ matrices. The quark masses and the $V_{C K M}$ matrix arise, for instance, using for the $d$-type quarks $a_{d}=0.14, h_{d}=h_{d}^{\prime}=0.1$, and (all VEVs and masses below are in $\mathrm{GeV}) v_{1}^{\prime \prime}=42, v_{2}^{\prime \prime}=1.4, v_{3}^{\prime \prime}=0.062, v_{\phi_{1}}^{\prime \prime}=2.552, v_{\phi_{2}}^{\prime \prime}=0.0074$, and $v_{\phi_{3}}^{\prime \prime}=11.83$ we obtain the masses: $m_{d}=0.006, m_{s}=0.1, m_{b}=4.2$. For the $u$-type quarks we use, $a_{u}=a_{d}$, but now $h_{u}=h_{u}^{\prime}=3$, and $v_{1}^{\prime}=$ 
$53.8, v_{2}^{\prime}=0.4, v_{3}^{\prime}=0.0001$ and $v_{\phi_{1}}^{\prime}=0.1, v_{\phi_{2}}^{\prime}=124, v_{\phi_{3}}^{\prime}=0.082$, which give $m_{u}=0.0025, m_{c}=1.27$, and $m_{t}=171.2$. With these VEVs and parameters we obtain $V_{L}^{D, U}$ matrices given, according with the definition above, the mixing matrix

$$
\left|V_{C K M}\right|=\left(\begin{array}{lll}
0.97747 & 0.21105 & 0.00148 \\
0.21083 & 0.97673 & 0.03923 \\
0.00972 & 0.03803 & 0.99923
\end{array}\right) .
$$

Comparing the elements of the matrix above with experimental data in Section 11.2 of PDG [25], we see that all the matrix elements, but us and $u b$ entries, are within the level of confidence (1-2) $\sigma$. However, this is enough to show that a realistic mixing matrix may arise from the quark mass matrices given in Eqs. (6) and (77). A more rigorous and detailed analysis is being done and will be presented later.

The suppression of the FCNCs coupled to neutral Higgs bosons can be obtained not naturally in the sense of Ref. [26], but at least as a reasonable fine tuning among the mixing matrix in the neutral scalar sector, $U_{i j}$, or by the mass of heavy Higgs scalars. For instance, consider the neutral kaons mass difference, $\Delta M_{K} \sim \zeta_{s d}^{i} f_{K}^{2} m_{K}\left(\sum_{j}\left|U_{i j}\right|^{2} / m_{j}^{2}\right)$ where $\zeta_{s d}^{i}=h_{d}^{2}\left|\sum_{a}\left(V_{L}^{D}\right)_{a s}^{*}\left(V_{R}^{D}\right)_{a d}\right|^{2}$, with symmetry eigenstates, say $H_{i}^{\prime \prime}$ ( $i$ fixed), and physical scalars $h_{j}$ with mass $m_{j}$. In order to be consistent with data $\zeta_{s d}^{i}$ must be smaller than the contribution of the SM: i.e., smaller that $G_{F}^{2} m_{c}^{2}\left(V_{c d}^{*} V_{c s}\right)^{2} / 16 \pi^{2}=0.5 \times 10^{-8} \mathrm{GeV}^{-2}$ (for simplicity we have taken into account only the $c$ quark contribution and neglect the QCD and $m_{c}^{2} / M_{W}^{2}$ corrections). Notice that the sum over the contributions of each $i$ is made coherently. The expression above is valid for any scalar heavier than $m_{K}$. For instance, $h_{d}^{2}\left|\sum_{a}\left(V_{L}^{D}\right)_{a s}^{*}\left(V_{R}^{D}\right)_{a d}\right|^{2} \sim 10^{-4}-10^{-6}$ and $\left|U_{i j}\right|^{2} / m_{j}^{2} \sim\left(10^{-2}-10^{-4}\right) \mathrm{GeV}^{-2}$ we have the appropriate FCNC suppression. These constraints are not a large fine tunings since it can be satisfied with a large set of unitary matrices and/or the neutral scalar mass spectrum and mixing. Recall that there is no more freedom to redefine the quark phases since they all have already been used to put the CKM matrix in the usual form, hence $V_{R}^{D}$ has five physical phases. The case of FCNCs in the $u$-quark sector involve the matrices $V_{L}^{U}$ and $V_{R}^{U}$.

On the other hand, it would be interesting if we can show that appropriate hierarchies among the VEVs arises from an analysis of the scalar potential [27]. We have verified that, under reasonable assumptions on the parameters in the scalar potential, this is indeed the case. For the sake of simplicity we will consider the scalar potential involving only the scalar doublets that couple to quarks: the $A_{4}$ triplets $H^{\prime}, \Phi^{\prime}$ for $u$-like quarks, and $H^{\prime \prime}, \Phi^{\prime \prime}$ for $d$-like quarks. Denoting $X_{u}=H^{\prime \dagger} H^{\prime}$ and $Y_{u}=\Phi^{\prime \dagger} \Phi^{\prime}, X_{d}=H^{\prime \prime \dagger} H^{\prime \prime}$ and $Y_{d}=\Phi^{\prime \prime \dagger} \Phi^{\prime \prime}$ we 
write the most general scalar potential invariant under the SM and $A_{4} \otimes Z_{3} \otimes$ $Z_{3}^{\prime} \otimes Z_{3}^{\prime \prime}$ symmetries as:

$$
\begin{aligned}
\mathcal{V} & =\mu_{p}^{2}\left[X_{p}\right]_{1}+\mu_{p}^{\prime 2}\left[Y_{p}\right]_{1}+\mu_{\zeta}^{2}|\zeta|^{2}+\lambda_{\zeta}|\zeta|^{4}+\left(\mu_{H^{\prime} \Phi^{\prime \prime}}^{2}\left[H^{\prime} \epsilon \Phi^{\prime \prime}\right]_{1}+\mu_{H^{\prime \prime} \Phi^{\prime}}^{2}\left[H^{\prime \prime} \varepsilon \Phi^{\prime}\right]_{1}\right. \\
& +f\left[\Phi^{\prime \dagger} H^{\prime}\right]_{1} \zeta+f^{\prime}\left[\Phi^{\prime \prime \dagger} H^{\prime \prime}\right]_{1} \zeta^{*}+f_{H}^{\prime}\left[H^{\prime \prime} \varepsilon H^{\prime}\right]_{1} \zeta+f_{\Phi}^{\prime}\left[\Phi^{\prime \prime} \epsilon \Phi^{\prime}\right]_{1} \zeta^{*} \\
& +\lambda_{m n}^{p p^{\prime}}\left[X_{p}\right]_{m}\left[X_{p^{\prime}}\right]_{n}+K_{k l}^{p p^{\prime}}\left[Y_{p}\right]_{k}\left[Y_{p^{\prime}}\right]_{l}+h_{\alpha \beta}^{p p^{\prime}}\left[X_{p}\right]_{\alpha}\left[Y_{p^{\prime}}\right]_{\beta}+g_{s t}\left[H^{\prime} \epsilon \Phi^{\prime \prime}\right]_{s}\left[H^{\prime} \epsilon \Phi^{\prime \prime}\right]_{t} \\
& \left.+g_{s t}^{\prime}\left[H^{\prime \prime} \varepsilon \Phi^{\prime}\right]_{s}\left[H^{\prime \prime} \varepsilon \Phi^{\prime}\right]_{t}+g_{s t}^{\prime \prime}\left(\left[H^{\prime} \epsilon \Phi^{\prime \prime}\right]_{s}\left[H^{\prime \prime} \varepsilon \Phi^{\prime}\right]_{t}\right)+H . c .\right) .
\end{aligned}
$$

We have omitted summation symbols and used the notation $p, p^{\prime}=u, d ; m n=$ $11,1^{\prime} 1^{\prime \prime}, A A ; k l=11,1^{\prime} 1^{\prime \prime}, B B ; \alpha \beta=11,1^{\prime} 1^{\prime \prime}, A B$; and $s t=11,1^{\prime} 1^{\prime \prime}, A A, B B, A B$. For example, $\lambda_{m n}^{p p^{\prime}}\left[X_{p}\right]_{m}\left[X_{p^{\prime}}\right]_{n}=\lambda_{11}^{p p^{\prime}}\left[X_{p}\right]_{1}\left[X_{p^{\prime}}\right]_{1}+\lambda_{1^{\prime} 1^{\prime \prime}}^{p p^{\prime}}\left[X_{p}\right]_{1^{\prime}}\left[X_{p^{\prime}}\right]_{1^{\prime \prime}}+\lambda_{A A}^{p p^{\prime}}\left[X_{p}\right]_{A}\left[X_{p^{\prime}}\right]_{A}$ $+\cdots$, and each term having contributions $p, p^{\prime}=u, d$. Considering the Eq. (12) we obtain the constraint equations arising from the conditions $\partial \mathcal{V} / \partial v_{i}^{\prime \prime}=0, \ldots$ For simplicity, in the following analysis it will be also assumed that the VEVs are all real. In this case the condition $1+\omega+\omega^{2}=0$ implies that in the constraint equations the coupling constants will appear as the sum of some of the coupling constants in Eq. (12). The inclusion of all the scalar fields of the model and also the constraints coming from the condition $\partial^{2} \mathcal{V} / \partial v_{i}^{\prime \prime} \partial v_{j}^{\prime \prime}>0, \ldots$ will be shown elsewhere. We have obtained the constraint equations under the following conditions: $v_{i}^{\prime \prime}=v_{\phi_{i}}^{\prime \prime}, \forall i$; and $\lambda_{m n}^{d d}=K_{k l}^{d d}, \forall m n ; k l$; and verified that, among the solutions to those equations, it is possible to have the following hierarchy: $v_{1}^{\prime \prime} \gg v_{3}^{\prime \prime} \gg v_{2}^{\prime \prime}$. However for generating the quark masses, as we have shown above, it is necessary that $v_{1}^{\prime(\prime \prime)} \gg v_{\phi_{1}}^{\prime(\prime)}$. Notwithstanding, it is interesting that such hierarchies can arise from the model, and we hope that a more detailed treatment of the scalar potential will produce a more realistic hierarchies among the VEVs.

As in any model with broken discrete symmetries there are potential troubles with domain walls [28]. However, this issue can be solved by allowing soft terms in the scalar potential violating these symmetries.

In conclusion, the mass matrices obtained, Eqs. (5), (6) , (7)), and (8) , arising because of the symmetries of the model, give appropriate insight concerning the solution of the flavor problem generating at the same time a realistic $V_{C K M}$ matrix. Of course, it is necessary to explain how these symmetries are realized from a more fundamental theory. Our scheme has some similarities with the "private Higgs" of Porto and Zee [29] in which there is one Higgs per fermion and a general mass matrices. However, we have more than one Higgs per fermion and the mass matrices have a specific texture of zeros.

We thank an anonymous referee for useful comments. ACBM was fully supported and VP partially supported by CNPq. 


\section{References}

[1] R. R. Volkas, Phys. Rev. D 53 (1996) 2681.

[2] M. C. Gonzalez-Garcia and M. Maltoni, Phys. Rept. 460 (2008) 1.

[3] A. Zee and F. Wilczek, Phys. Rev. Lett. 42 (1979) 421.

[4] Y. Koide and A. Ghosal, Phys. Rev. D 63 (2001) 037301.

[5] H. Zang, Phys. Lett. B665 (2007) 132.

[6] H. Sawanaka, Int. J. Mod. Phys. E16 (2007) 1383, M. Hirsch, A. S. Joshipura, S. Kaneko and J. W. F. Valle, Phys. Rev. Lett. 99 (2007) 151802; R. R. Volkas, hep-ph/0612296 B. Adhikary, B. Brahmachari, A. Ghosal, E. Ma and M. K. Parida, Phys. Lett B638 (2006) 345; E. Ma, Mod. Phys. Lett. A20 (2005) 2601; Europhys. Lett. 79 (2007) 61001; Mod. Phys. Lett. A22 (2007) 101; and hep-ph/0606024; L. Lavoura and H. Kuhbock, Eur. Phys. J C55 (2008) 303.

[7] P. H. Frampton and T. W. Kephart, Int. J. Mod. Phys. A10 (1995) 4689.

[8] A. Aranda, C. D. Carone, R. F. Lebed, Phys. Lett. B474 (2000) 170; Phys. Rev. D 62 (2000) 016009; A. Aranda, C. D. Carone, and P. Meade, ibid. D 65 (2001) 013011; F. Feruglio, C. Hagedorn, Y. Lin, and L. Merlo, Nucl. Phys. B775 (2007) 120; M-C. Chen and K. T. Mahanthappa, Phys. Lett. B652 (2007) 34; P. H. Frampton and S. Matsuzaki, arXiv:0710.5928.

[9] K. S. Babu and J. Kubo, Phys. Rev. D 71 (2005) 056006; N. Kifune, J. Kubo, and A. Lenz, Phys. Rev. D 77 (2008) 076010.

[10] P. H. Frampton and T. W. Kephart, JHEP 0709 (2007) 110; C. Luhn, S. Nasri, and P. Ramond, J. Math. Phys. 48 (2007) 073501; ibid (2007) 123519; and Phys. Lett. B652 (2007) 27.

[11] S. Weinberg, Phys. Rev. Lett. 43 (1979) 1566; F. Wilczek and A. Zee, Phys. Rev. Lett. 43 (1979) 1571.

[12] A. Zee, Phys. Lett., B93 (1980) 389; ibid, B95 (1980) 461(E).

[13] E. Ma and G. Rajasekaran, Phys. Rev. D 64 (2001) 113012; E. Ma, Int. J. Mod. Phys. A23 (2008) 3366; P. D. Carr and P. H. Frampton, hep-ph/0701034.

[14] K. S. Babu, E. Ma, and J. W. F. Valle, Phys. Lett B552 (2003) 207; E. Ma, hep-ph/0606024; H. Sawanaka, Int. J. Mod. Phys. E16 (2007) 1383.

[15] G. Altarelli and F. Feruglio, Nucl. Phys. B741, 215 (2006); G. Altarelli, hep-ph/0611117.

[16] P. F. Harrison, D. H. Perkins, and W. G. Scott, Phys. Lett. B349 (1999) 137; ibid B458 (1999) 79; ibid B530 (2002) 167.

[17] X-G. He, Y-Y. Keum, and R. R. Volkas, JHEP 0604 (2006) 039. 
[18] T. P. Cheng and L-F. Li, Phys. Rev. D 22 (1980) 2860.

[19] H. Fritzsch and Z. Z. Xing, Prog. Part. Nucl. Phys. 45 (2000) 1.

[20] J. C. Montero, C. A. de S. Pires, and V. Pleitez, Phys. Rev. D 60 (1999) 098701, M-C. Chen, S. Dawson, and T. Krupovickas, Phys. Rev. D 74 (2006) 035001, and references therein.

[21] B. Brahmachari and S. Choubey, Phys. Lett. B531 (2002) 99.

[22] B. Brahmachari and S. Choubey, Phys. Lett. B642 (2002) 495.

[23] M. Appolonio et al. (CHOOZ Collaboration), Phys. Lett. B420 (1998) 415; ibid B466 (1999) 397.

[24] T. V. Duong and E. Ma, Phys. Rev. D 47 (1993) 2020; T. V. Duong, E. Keith, E. Ma, and H. Kikuchi, ibid. D 52 (1995) 5045.

[25] C. Amsler et al., Particle Data Group, Phys. Lett. B667 (2008) 1.

[26] S. L. Glashow and S. Weinberg, Phys. Rev. D 15 (1977) 1958.

[27] A. Zee, Phys. Lett. 630B (2005) 58.

[28] Y. B. Zeldovich, I.Y. Kobzarev, and L.B. Okun, Sov. Phys. JETP 40 (1975) 1; P. Sikivie, Phys. Rev. Lett. 48 (1982) 1156.

[29] R. A. Porto and A. Zee, Phys. Lett. B666 (2008) 491. 